\title{
Does protein supplementation impact appetite and energy intake in older adults? A systematic review
}

\author{
Sana Ben-Harchache and Katy Horner \\ University College Dublin, Dublin, Ireland
}

\section{Abstract}

Protein supplementation represents an attractive strategy to prevent skeletal muscle mass loss and function with aging. However, it could be counterproductive in older adults, due to adverse effects on appetite and energy intake (EI). Understanding the effects of protein supplementation on appetite and energy intake is therefore key when investigating the potential efficacy for use in improving health outcomes. This systematic review aimed to assess the literature investigating the effects of protein supplementation on energy intake, and/or appetite in healthy older adults.

PubMed and The Cochrane Library, as well as MEDLINE, CINAHL, EBSCO, and Web of Science databases were searched up to 21 March 2019 for published studies and theses. The review was limited to randomized controlled trials and longitudinal studies in healthy adults over 60 years of age that reported effects of protein supplementation compared to control or pre-intervention on appetite and/or energy intake. The review was conducted according to PRISMA guidelines.

Out of 5,429 articles screened, 90 full-texts were reviewed and twenty-six studies (11 acute, 15 longitudinal) included. Studies involved a total of 1439 participants (502 male, 881 females). Of the acute studies, 3 reported conditions in which appetite was suppressed, 5 studies reported no effect and one study reported a condition in which appetite ratings increased following a protein load versus control. Three studies reported conditions in which ad libitum test meal EI was suppressed following protein preload and 7 reported no difference. When examining total EI (accounting for protein supplement and test meal), 2 studies reported no difference and 8 reported conditions where total EI was increased with protein supplement compared to control. Longitudinal studies ranged from 2-26 weeks duration. Two studies reported effects on appetite, one showing hunger to be suppressed on higher $(1.0 \mathrm{~g} / \mathrm{kg} / \mathrm{d})$ compared to lower protein diet, and another showing hunger in response to whey consumption increased, but fullness also increased.14 studies reported effects on EI, most commonly assessed by food diary, and all showed no effect of increased protein intake compared to control on daily EI.

The systematic review indicates that while appetite may be suppressed under some conditions, the current literature supports protein supplementation in older adults, as it either has a positive effect or no effect on total EI in both short and longer-term studies. Therefore, protein supplementation may represent an effective solution to address protein deficiencies in older adults without compromising daily EI.

\section{Conflict of Interest}

There is no conflict of interest. 